FORMATION Formation emploi

Revue française de sciences sociales

103 | juillet-septembre 2008

Fuite ou mobilité des cerveaux?

\title{
Le non-retour des étudiants étrangers : au-delà de la « fuite des cerveaux »
}

Non-return of foreign students: beyond the "brain drain"

Definitive Abwanderung ausländischer Studenten

El no retorno de los estudiantes extranjeros : más allá de la « fuga de cerebros »

\section{Ridha Ennafaa et Saeed Paivandi}

\section{(2) OpenEdition}

Journals

\section{Édition électronique}

URL : http://journals.openedition.org/formationemploi/2356

DOI : 10.4000/formationemploi.2356

ISSN : 2107-0946

\section{Éditeur}

La Documentation française

\section{Édition imprimée}

Date de publication : 1 septembre 2008

Pagination : 23-39

ISSN : 0759-6340

\section{Référence électronique}

Ridha Ennafaa et Saeed Paivandi, « Le non-retour des étudiants étrangers : au-delà de la « fuite des cerveaux » », Formation emploi [En ligne], 103 | juillet-septembre 2008, mis en ligne le 01 septembre 2010, consulté le 30 octobre 2020. URL : http://journals.openedition.org/formationemploi/2356 ; DOI : https://doi.org/10.4000/formationemploi.2356 


\title{
DOSSIER
}

\section{Le non-retour \\ des étudiants étrangers : au-delà de la «fuite des cerveaux»}

Ridha Ennafaa et Saeed Paivandi*

\author{
La mobilité internationale des étudiants sera-t-elle ou non \\ suivie d'un retour au pays, sachant que les projets d'avenir des \\ étudiants renvoient à une grande diversité de comportements fondés \\ sur des logiques professionnelle, migratoire, intégratrice ou existentielle?
}

La question du retour des étudiants étrangers dans leur pays revêt aujourd'hui un caractère particulier en France dans le contexte national, régional et international. Les pouvoirs publics tentent d'intégrer l'accueil des étudiants étrangers à la politique d'immigration choisie. Ce discours vis-à-vis des étudiants étrangers est effectivement nouveau, mais la réflexion sur le sujet est assez ancienne. En effet, depuis les années 60, le phénomène de fuite des cerveaux a fait couler beaucoup d'encre à travers le monde. La recherche française s'intéresse essentiellement, à partir des années 80 , à la question du retour des étudiants étrangers et à l'ampleur du phénomène de la fuite des cerveaux en France.

La France se situe parmi les quatre premières destinations des étudiants étrangers au niveau mondial. Selon les données de l'OCDE (2006), en 2004, avec 572509 inscrits, les États-Unis ont accueilli près de $22 \%$ des étudiants étrangers dans le monde, suivis par l'Angleterre (11\%), l'Allemagne (10\%) et la France $(9 \%)$. Malgré la domination américaine, la part relative des étudiants étrangers inscrits dans ce pays ne cesse de diminuer ( $32 \%$ en 1985 et $25 \%$ en 2000). Au niveau mondial, au cours des deux dernières décennies, le nombre de jeunes poursuivant des études supérieures (universitaires et non universitaires) dans un pays étranger a été multiplié par 2,5 : ils étaient près d'un million en 1985, et plus de 2,6 millions en 2004. Selon ces données, plus de $85 \%$ des étudiants étrangers dans le monde s'inscrivent dans l'un des établissements d'enseignement

* Ridha Ennafaa et Saeed Paivandi sont maîtres de conférences à l'université Paris 8 et membres de l'équipe ESSI (Education, Socialisation, Subjectivation, Institution) CRES (Centre de recherche sur l'enseignement supérieur). Ils ont publié, avec A. Coulon, un ouvrage intitulé Devenir enseignant du supérieur (L'Harmattan, 2004). Leur ouvrage sur l'enquête de 2005 de l'OVE (Observatoire de la vie étudiante) sur les étudiants étrangers intitulé Venir faire des études en France paraîtra en septembre 2008 à La Documentation française. 


\section{Encadré 1 \\ Une enquête sur 1715 étudiants étrangers en France en 2005}

L'enquête de l'OVE (Observatoire de la vie étudiante) sur les étudiants étrangers en France a été réalisée entre avril et juillet 2005 auprès d'un échantillon représentatif constitué à partir des fichiers d'inscription des étudiants étrangers en mobilité (venus en France pour faire des études; les résidents étrangers ont été exclus). Dix universités de Paris et région parisienne (Paris 1, 3, 5, 6, 8 et 12) et en province (Nantes, Strasbourg 1, Lyon 2 et Nice) sélectionnées dans l'ensemble des universités françaises selon trois critères (origine géographique des étudiants étrangers, leur niveau et discipline d'études en 2004-2005) ont participé à l'enquête. Un échantillon de 1715 étudiants établi selon ces trois quotas a répondu à un questionnaire de plus de cent questions administré " en face-à-face » par une équipe d'enquêteurs. 35 entretiens post-enquête ont permis de mieux étoffer certaines analyses.

L'enquête s'est centrée sur les conditions de vie et d'études des étudiants étrangers ainsi que sur leurs parcours universitaires et leurs projets. Les réponses de l'enquête, ainsi qu'une série d'une quarantaine d'entretiens (postenquête), ont permis de dresser un tableau des éléments de réponses sur ce qu'ils pensaient faire après leurs études à travers leur expérience en cours.

Nous avons réparti les étudiants selon sept grandes régions d'origine. Près d'un étudiant sur trois était ainsi originaire du Maghreb (Algérie, Maroc, Tunisie), et 21 \% de notre échantillon provenaient des autres pays africains (essentiellement de l'Afrique subsaharienne). Un même pourcentage rassemblait les étudiants européens $111 \%$ venant de l'Union européenne et $10 \%$ des autres pays européens), tandis que $12 \%$ étaient originaires d'Asie de l'Est (tous les pays de l'Est et du Sud-Est du continent, à partir de I'Inde), $7 \%$ du Proche et du Moyen-Orient (de l'Asie Centrale à l'Égypte) et $6 \%$ des Amériques (Amérique du Nord ou Amérique Latine). Cette répartition se révèle relativement proche de celle de la population étrangère inscrite dans les universités françaises en 2004-2005 (*). Les femmes constituaient un peu plus de la moitié de l'échantillon, les plus nombreuses d'entre elles provenant d'Europe ou d'Asie, tandis que les hommes s'avéraient particulièrement sur-représentés chez les Africains (sub-sahariens), les Maghrébins, et les étudiants originaires du Moyen-Orient.

En termes de niveau d'études, $22 \%$ étaient inscrits dans les deux premières années du cycle universitaire (Deug 1 -diplôme d'études universitaires générales -, Deug 2, L1, L2, DUT 1 - diplôme universitaire de technologie -, DUT 21, 19,5\% en licence ou L3, 21 \% en maîtrise ou Master 1, 13 \% en DEA Diplôme d'études approfondies -, DESS - diplôme d'études supérieures spécialisées - ou Master 2, et $15,5 \%$ en doctorat $\left(^{* *}\right)$. Un étudiant sur trois suit une filière "scientifique et technique », un peu moins d'un sur cinq choisit les "sciences sociales et humaines », $24 \%$ sont inscrits dans les disciplines " juridiques ou économiques» et 16,5\% en « lettres, arts et langues ».

(*) : Selon la DEPP (MEN), plus de $51 \%$ des étudiants étrangers inscrits dans les universités en France en 2004-2005 viennent des pays d'Afrique, $24 \%$ d'Europe, $18 \%$ d'Asie, et $7 \%$ d'Amérique (DEPP, Repères et références statistiques, 2005).

$(* *)$ : Selon la DEPP (MEN), la population étrangère représente $11 \%$ des étudiants inscrits dans le cursus de licence. Elle représente $19 \%$ pour le niveau master et $35 \%$ pour le doctorat.

supérieur des pays de l'OCDE (Organisation de coordination et de développement économiques), et $70 \%$ d'entre eux sont originaires des pays dits « du Sud» (hors pays de l'OCDE). La France a connu une évolu- tion importante depuis 1990 : une diminution de cette population au début des années 90 , suivie, à partir de 1997, d'une augmentation continue, qui s'est accentuée à partir de 1999. De 1999 à 2004, les effectifs des 
étudiants étrangers dans l'enseignement supérieur français ont crû au total de 59 \% ${ }^{1}$. En 2004-2005, plus de $11 \%$ des étudiants inscrits (255 585 personnes) dans les différents établissements supérieurs (14\% dans les universités avec 202836 inscrits) sont étrangers, contre seulement $7 \%$ en 1998.

L'article vise à examiner la question du projet d'avenir des étudiants étrangers en s'appuyant sur les résultats de l'enquête de l'OVE (Observatoire de la vie étudiante) national réalisée en 2005 sur un échantillon représentatif de 1715 étudiants étrangers en France. Il s'agit d'examiner la façon dont les étudiants abordent la question du retour dans leur pays d'origine en fonction de leurs caractéristiques. L'intérêt de cette enquête repose essentiellement sur son caractère transversal (disciplines et cycles d'études) et l'étendue de son échantillon (l'origine géographique des étudiants). Elle a été réalisée à un moment particulier, marqué à la fois par la politique d'ouverture tous azimuts voulue par les autorités à partir de 1998, et par la mise en place d'un dispositif filtrant $(\mathrm{CEF})^{2}$ pour mieux cibler les étudiants étrangers.

L'article est composé de deux parties : la première explore le concept de « fuite des cerveaux », son lien avec les études à l'étranger et son évolution depuis les années 60, dans une perspective internationale. La deuxième partie est consacrée à l'analyse du projet d'avenir des étudiants étrangers en France, à partir des résultats de l'enquête de 2005 de l'OVE.

\section{LES ÉTUDES À L'ÉTRANGER ET LA FUITE DES CERVEAUX}

Le paradigme de la " fuite des cerveaux», appelé aussi «exode des compétences» ou «exode des cerveaux », développé à partir des années 60 , tentait d'expliquer et d'analyser les flux migratoires des personnes qualifiées à travers le monde dans le cadre

\footnotetext{
${ }^{1}$ Toutes les données sur les étudiants étrangers en France sont issues des publications de la DEP/MEN.

${ }^{2}$ Centre pour les études en France (CEF) mis en place par le ministère des Affaires étrangères dans plusieurs pays comme la Chine, la Tunisie, l’Algérie, le Maroc, le Vietnam et le Sénégal. Ce dispositif est destiné à vérifier, sur les plans linguistique, financier et scientifique, le dossier des candidats aux études en France.
}

des théories du capital humain ${ }^{3}$. La lecture des publications de la période 1960-1980 sur ce phénomène permet d'identifier deux thèses récurrentes :

La première concerne le lien direct entre les études à l'étranger et le développement de la fuite des cerveaux. C'est effectivement durant cette période que certains pays ont commencé à réaliser qu'une partie des étudiants expatriés ne rentraient pas chez eux à la fin des études. Les études à l'étranger deviennent de facto l'un des canaux migratoires à travers le monde. Une étude récente dresse un bilan global depuis 1960 : la mobilité étudiante représente un flux potentiel de travailleurs qualifiés, soit pendant le temps de leurs études, soit sous forme de recrutement ultérieur; ainsi, les flux d'étudiants constituent une forme de migration des travailleurs qualifiés et un précurseur de migrations ultérieures (OCDE, 2002).

La deuxième thèse aborde le phénomène de la fuite des cerveaux comme un mouvement unidirectionnel. Le brain drain ${ }^{4}$ devient descripteur des flux unilatéraux de la «Périphérie » vers « le Centre » (Meyer et Charum, 1995). Autrement dit, les étudiants étrangers venant du Sud ont plus de probabilité que ceux des pays avancés de s'établir définitivement dans leur pays d'accueil. L'UNESCO avait fait ce constat dès le début des années 60 : "on croit généralement que les pays sous-développés auxquels font cruellement défaut les cadres nécessaires à leur développement reçoivent une assistance technique des pays riches. En réalité, c'est le contraire qui se passe. Les chiffres prouvent que ce sont les pays sousdéveloppés qui, chaque année, fournissent des médecins, des ingénieurs, des professeurs, aux pays industriels. » (Naraghi, 1965) Cette thèse est confirmée par de multiples recherches: Meyer et Hernandez (2004) se réfèrent aux différentes données pour qualifier les études à l'étranger de « voie royale de l'expatriation » dans la mesure où la majorité des immigrés hautement qualifiés sont entrés dans le

\footnotetext{
${ }^{3}$ Les différentes théories du capital humain mettent en relation l'ensemble de connaissances, d'aptitudes et de compétences acquises par les individus avec la productivité économique et la création d'un bien-être personnel, social.

${ }^{4}$ Le terme de «brain drain » a été initialement utilisé dans les années 50 pour décrire l'exode de scientifiques et d'ingénieurs britanniques vers les États-Unis et le Canada.
} 
pays d'accueil en tant qu'étudiants. Les auteurs estiment que c'est le cas pour les deux tiers d'entre eux, avec des différences selon les pays.

De nombreux travaux universitaires pionniers, essentiellement aux États-Unis, s'intéressent à cette question en cherchant à élaborer un modèle explicatif au phénomène de la fuite des cerveaux en lien avec le rôle joué par les études à l'étranger. Les recherches se multiplient pour mieux cerner son ampleur et ses mécanismes. Celles qui s'intéressent aux raisons du non-retour (ou retour) des étudiants étrangers identifient deux types de facteurs «explicatifs»: les facteurs d'attraction (pull factors) d'une part, qui influencent la décision des étudiants avant et pendant le séjour éducatif (salaire, conditions de vie ou de travail); les facteurs «centrifuges» (push factors) d'autre part, comme les difficultés de réinsertion dans le pays d'origine ou les situations particulières (guerre, crise politique ou économique) motivant les décisions du non-retour ou du retour (Bhagwati, 1976 ; Glaser, 1973 et 1978 ; Gossman et al., 1968).

Le modèle théorique économique (coûts et bénéfices) adopté par la plupart des recherches de cette période semble être influencé par le contexte américain. L'étudiant étranger dans un système universitaire payant est considéré comme un acteur disposant d'un espace de décision variable selon sa position sociale. Il est doté d'une rationalité qui lui fait évaluer à chaque moment important de son parcours universitaire les coûts et les avantages de la poursuite des études. Une des visions la plus présente du brain drain est fondée sur une perspective macroéconomique qui cherche à établir une similitude entre les flux internationaux de biens et de services et la mobilité des ressources intellectuelles. Pour Agarwal et Winkler (1985b), la décision relative à l'immigration est prise si le coût de cet acte reste inférieur au bénéfice qui en est attendu. Selon Salomon, le talent irait où il est le mieux rémunéré ou « reconnu » (1991).

Agarwal et Winkler (1985a) ont également tenté, dans les années 70 et 80 , de chiffrer ce phénomène et d'examiner en même temps le rôle joué par les mesures (la possibilité de modifier le statut étudiant pour obtenir une autorisation de séjour en tant que travailleur) mises en place pour favoriser l'instal- lation des étudiants étrangers aux États-Unis. Les auteurs évoquent l'immigration «indirecte» aux États-Unis pratiquée par les étudiants étrangers qui réussissent à modifier leur statut initial : de détenteurs d'un visa étudiant, ils deviennent des résidents. Les auteurs établissent un bilan chiffré et détaillé de la période 1965-1985. Cet inventaire est révélateur : une partie des étudiants étrangers, variable selon les pays, préfère s'installer aux États-Unis et leur poids reste marginal, sauf si on les compare aux migrants ayant déjà obtenu un diplôme supérieur dans leur pays d'origine.

Par ailleurs, Agarwal et Winkler (1984) notent que les études sur l'immigration professionnelle ignorent souvent le cadre légal comme un facteur influent. Selon cette recherche, la politique américaine en matière d'immigration a un impact indéniable sur le changement de statut des étudiants, et ceci varie selon leur pays d'origine. Les auteurs concluent que si l'on veut réduire la fuite de cerveaux, il faut changer de politique d'accueil en fonction des pays d'origine.

\section{Un nouveau discours dans un nouveau contexte}

Vers la fin des années 80 , on note une évolution du discours sur les étudiants étrangers, en rapport avec l'exode des cerveaux. Dans un contexte marqué par l'accélération du processus de la mondialisation et par l'intensification des flux migratoires, le paradigme de la fuite des cerveaux est de plus en plus perçu comme inadéquat pour expliquer toutes les dimensions de ce phénomène et sa complexité. Les chercheurs tendent à exprimer que la migration des cerveaux ne représente pas toujours et irrémédiablement une perte pour les pays d'origine (Gaillard et Gaillard, 1999 ; Nedelcu, 2004 ; Meyer et Charum, 1985). L'utilisation des nouveaux termes comme brain gain (le gain de cerveaux), transit brain drain (l'exode de cerveaux transitaire) ou delayed return (le retour différé) ou encore brain mobility (la mobilité des cerveaux) témoignent de ce changement contextuel.

L'émergence de ce nouveau discours sur le phénomène du non-retour des étudiants étrangers ou sur l'exode des cerveaux coïncide avec plusieurs 
évolutions liées au contexte économique, technologique et éducatif sur le plan international. Les mutations technologiques remettent en question les notions de distance et de frontière et entraînent des comportements et des stratégies de mobilité très variés, en particulier chez les plus jeunes (Nedelcu, 2004, p. 10). Selon l'auteur, un véritable marché international des compétences fonctionne dans certains secteurs très spécialisés de l'économie internationale. Dans ce contexte, les pays qui sont confrontés à une pénurie de main-d'œuvre très qualifiée dans ces secteurs sont de plus en plus tentés par une politique migratoire sélective. De même, de nombreux pays ont introduit récemment d'importants ajustements concernant les possibilités de changement de statut et d'accès au marché du travail pour les étudiants étrangers en fin de formation. La politique migratoire américaine est considérée comme l'un des éléments-clés de l'expansion économique de ce pays puisqu'elle a permis l'accroissement et le renouvellement du potentiel en ressources humaines hautement qualifiées en sciences et en technologie (Gaillard et Gaillard, 1999). Le rapport de l'observatoire des migrations de l'OCDE (2006) note ainsi que les migrations traditionnelles de travail sont de plus en plus limitées en faveur de l'admission temporaire des travailleurs qualifiés. Ce rapport soutient que la mobilité étudiante demeure une voie importante des tendances migratoires.

Un des points souvent évoqués dans l'évolution de la population étudiante dans les pays du Nord est la désaffection accrue à l'égard des études scientifiques et le manque effectif de candidats pour ces filières, $y$ compris au niveau du troisième cycle. C'est ainsi que la mobilité internationale des étudiants prend une dimension importante du développement de l'enseignement supérieur et devient un enjeu majeur de la compétition au niveau mondial, aussi bien sur le plan économique que sur le plan scientifique et culturel. Ces changements ont engendré une situation inédite caractérisée par une compétition internationale ouverte pour attirer les meilleurs étudiants et chercheurs. Les pays qui sont considérés comme les pays d'accueil peuvent devenir en même temps les pays de départ des jeunes chercheurs. Autrement dit, l'exode des cerveaux n'affecte plus uniquement les pays du Sud ; le mouvement s'étend vers les pays du
Nord. On repère, par exemple, le départ des étudiants avancés du Canada ou de l'Europe vers les ÉtatsUnis (Cervantes et Guellec, 2002). ${ }^{5}$

Par ailleurs, certains pays exportateurs de « cerveaux » semblent prendre conscience de l'enjeu et de la complexité des migrations au cours de ces dernières décennies. C'est surtout le cas des pays émergents, considérés autrefois comme "perdants» dans la course mondiale aux talents, qui s'organisent pour mettre en place des dispositifs visant à réduire leur départ ou à favoriser leur réintégration. Cette stratégie, mise en place par les grands " exportateurs » d'étudiants vers les pays du Nord, comme l'Inde, la Chine, la Corée du Sud, certains pays d'Amérique du Sud ou d'Afrique du Sud, est facilitée par la constitution de réseaux de scientifiques expatriés qui se mobilisent et institutionnalisent les transferts technologiques et d'expertises vers leur pays d'origine. Une nouvelle vision de la diaspora fondée sur une sorte de «transnationalisme » émerge, et certains pays commencent à s'ouvrir aux nouvelles perspectives en matière de mobilité internationale des étudiants et des chercheurs. Selon Meyer et Charum (1995), la fuite des cerveaux irrigue des réseaux qui regroupent, sur des bases nationales ou régionales, des expatriés originaires de pays en développement. Ceux-ci contribuent à distance à la construction d'un milieu scientifique dans leurs pays d'origine en accueillant par exemple des étudiants ou des collègues, en faisant circuler des informations bien ciblées grâce aux TIC (technologies de l'information et de la communication), etc. En se référant à cette analyse, les auteurs considèrent que le modèle du brain drain, qui servit à décrire le phénomène du non-retour durant les dernières décennies, est épuisé.

Cette nouvelle donne migratoire semble influencer le comportement des étudiants étrangers qui sont confrontés à la question du retour. L'expansion de la liberté de circulation rend le retour possible à tout moment, les départs des étudiants et diplômés ne sont

\footnotetext{
${ }^{5}$ Les auteurs notent le projet initié en 2000 par le gouvernement britannique et la fondation de recherche Wolfson, visant à encourager des scientifiques britanniques de haut vol à revenir au pays, et à attirer au Royaume-Uni de jeunes chercheurs du monde entier. On peut mentionner également l'opération «Rientro die cervelli » lancée par le gouvernement italien pour lutter contre l'exode des étudiants avancés et des chercheurs (Perna et Carles, 2004).
} 
pas à sens unique et ils gardent des attaches réelles ou symboliques. Nedelcu (2004) parle d'un phénomène émergeant de «globalocalisation» qui se manifeste par la capacité des migrants à se positionner en tant que médiateurs sociaux et culturels dans plusieurs localités à la fois, grâce à la multiplicité des appartenances, des légitimités, et au potentiel grandissant des mises en réseau. Le rapport de Vuilletet, élaboré à la demande du Conseil économique et social (2005), examine ce changement paradigmatique. Le texte mentionne les intérêts économiques, culturels, scientifiques et stratégiques de l'accueil des étudiants étrangers en France dans une perspective comparative, tout en considérant que ceci constitue également un atout pour les pays d'origine. Tout tend à montrer qu'avec le changement du contexte, le brain gain renvoie à une lecture critique de l'exode des cerveaux Sud-Nord dans le domaine des migrations scientifiques (Michaelis, 1990; Gaillard et Gaillard, 1999). Cette notion exprime la possibilité de rapatriement de compétences pour favoriser le développement national. C'est dans cette perspective que la population qualifiée expatriée peut être considérée comme un avantage potentiel plutôt que comme une perte sèche et définitive.

Cependant, une approche critique des différentes théories explorant les formes multiples de la mobilité internationale des étudiants et chercheurs invite à la prudence. Le processus qui permet de transformer l'exode des cerveaux en mouvement de migration, de mobilité ou de circulation ne va pas de soi. L'émigration de «cerveaux» ou leur circulation sont largement conditionnées par la capacité de chaque pays à récupérer les talents qui se sont formés à l'extérieur. Dans cette perspective, la fuite massive de cerveaux continue à toucher les pays les plus vulnérables du Sud (Boussichas, 2005), comme on peut également le lire dans un rapport récent de la Banque mondiale (2004).

Deux exemples dans ce domaine peuvent illustrer la coexistence de situations contrastées. Au seuil des années 60 , un grand nombre de Coréens restaient à l'étranger une fois formés (par exemple, c'était le cas aux États-Unis pour $80 \%$ d'entre eux). Or, la décennie 80 a vu s'inverser cette tendance avec le retour des deux tiers des titulaires de doctorats américains dans les trois années qui suivaient l'obtention de leur diplôme. En revanche, les pays maghrébins, le Liban ou l'Iran, qui avaient envoyé proportionnellement un nombre élevé d'étudiants à l'étranger, ne connaissent pas ce mouvement de retour. Wickramasekara (2003) relève deux facteurs qui semblent favoriser le retour des étudiants originaires de la Corée du Sud et de Taiwan formés à l'étranger : la croissance économique locale, des politiques gouvernementales actives et les mesures incitatives destinées aux personnes ayant terminé leurs études à l'étranger. Par exemple, les diplômés des universités étrangères sont accompagnés et aidés dans la recherche d'emploi ou une aide financière de « réinstalation » leur est accordée.

\section{La recherche française et la question du non-retour}

Comparée à l'Amérique du Nord, la recherche française sur les étudiants étrangers est restée très peu développée. Il faut attendre la fin des années 80 pour voir se développer des travaux de recherches qui s'y intéressent, abordant essentiellement le cas des étudiants du maghreb et de l'afrique sub-saharienne. Tout d'abord, les recherches françaises tendent à confirmer que les étudiants étrangers originaires des pays du Sud sont souvent devant une double perspective : réinsertion dans les pays d'origine ou sédentarisation en France ou dans un autre pays. Les étudiants évoquent les raisons les poussant à décider de rester en France: la pauvreté des universités locales, le faible niveau technologique et l'insuffisance chronique des infrastructures, l'accès difficile à l'information, l'absence de moyens et de programmes de recherche, la désertification culturelle, la corruption, le sous-développement chronique, les crises politiques et économiques, la dégradation du cadre de vie (Borgogno et Vollenweider-Andresen, 1998 ; Sefrioui, 1997 ; Latrèche, 1999 ; Paivandi, 1991, Ben Sedrine et Geisser, 2001).

Les plus exposés au phénomène du non-retour sont les étudiants avancés. Selon l'OCDE, le taux de nonretour des étudiants docteurs est très similaire d'un pays du Nord à un autre (OCDE, 1998). Rovet, Terouanne et Neher (1998) révèlent que seulement la moitié des étudiants étrangers titulaires d'un doctorat ou d'un post-doc en France retournent dans les deux 
ans dans leur pays. Le rapport de Raunet (2001) estime qu'au moins un tiers des étudiants étrangers finalisant leur doctorat sur le territoire resterait en France. Concernant les étudiants marocains inscrits dans les Grandes Écoles, le taux de retour serait de l'ordre de $60 \%$.

Plusieurs enquêtes réalisées depuis la fin des années 80 mettent en évidence l'ampleur de ce phénomène. Selon Borgogno et Vollenweider-Andresen (1998), chez les Maghrébins, seulement près d'un étudiant sur deux se dit décidé à rentrer au pays, et plus de $40 \%$ sont dans l'incertitude quant à leur avenir. Les auteurs avancent deux raisons qui compromettent l'hypothèse du retour: la première est professionnelle et économique. La deuxième raison est culturelle, les étudiants anticipant les problèmes que leur paraît poser leur réintégration dans le cadre social et culturel de leur pays d'origine. Dans l'enquête de Latrèche, $28 \%$ des étudiants maghrébins déclaraient ne pas vouloir retourner dans leur pays d'origine (1999, p. 201). Sefrioui (1997) montre qu'entre 1992 et 1993, un peu plus de la moitié des 569 étudiants de son enquête ayant terminé leur thèse sont rentrés dans leur pays d'origine et près de $30 \%$ sont restés en France. Selon l'auteur, face à la crise économique et politique qui touche certains pays en développement, la stratégie individuelle dominante des études à l'étranger risque de se confondre avec une volonté d'émigration.

Par ailleurs, une étude algérienne, réalisée auprès des étudiants algériens en France, en Grande-Bretagne, au Canada et aux États-Unis, permet de dresser un constat fort révélateur: "face au double abandon scientifique et professionnel dont ils sont victimes, les étudiants algériens à l'étranger sont tentés soit de prolonger la durée de leurs études $(37 \%$ des étudiants interrogés ont demandé une prolongation), soit de ne pas revenir- sur 700 boursiers qui devaient rentrer en 1991, 50 seulement l'ont fait.» (Benguerna et Khelifaoui, 1993)

Les enquêtes sur les étudiants maghrébins sont souvent posées en des termes qui prennent en compte la spécificité de la présence des étudiants du Maghreb dans les universités françaises.

Les bourses d'études accordées par la France, l'aide fournie aux établissements supérieurs ou l'action des établissements secondaires français dans les pays africains sont ainsi l'objet de multiples critiques. Orivel (1991, p. 381) fait la même remarque en considérant que le rôle de la France «qui aide de nombreux pays francophones à combler leur déficit chronique en enseignants de mathématiques, détruit, involontairement ou non, son oeuvre en recrutant elle-même, pour ses propres besoins, une proportion significative de ces diplômés ».

Le cas des autres pays en situation de crise est également révélateur. Les résultats obtenus lors de l'enquête de Paivandi (1991, p. 385) sur les Iraniens sont frappants : seulement $12,5 \%$ des personnes interrogées pensent rentrer rapidement en Iran après la fin de leurs études, contre $39 \%$ qui ont l'intention de travailler en France, et $48,5 \%$ qui ne le savent pas encore.

Un point commun entre les recherches françaises et américaines concerne les difficultés liées à la réadaptation dans le pays d'origine une fois les études achevées, et leur impact sur la décision du non-retour. Selon Behnam (1981, p. 11), en rentrant chez lui, l'étudiant doit faire face à un difficile exercice de réadaptation: le "transfert des connaissances et l'essai de synthèse entre les valeurs étrangères et indigènes ». En témoignent les nombreux exemples de scientifiques ou d'ingénieurs talentueux, mal ou sous-utilisés lorsqu'ils retournent dans leur pays, où leurs compétences sont déconnectées de ce qui les rendaient performants (Gaillard et Gaillard, 1999).

Dans une perspective plus socioculturelle, S. Kim (2000) souligne l'importance du problème du « retour» des étudiantes asiatiques dans leur pays d'origine compte tenu de l'acculturation engendrée par la culture du pays d'accueil. S. Paivandi (1991) évoque les mêmes raisons pour les étudiantes iraniennes pour lesquelles les études à l'étranger ont un impact «émancipateur».

Que peut-on dire aujourd'hui à propos du phénomène du non-retour des étudiants étrangers dans le contexte français? Les nouvelles politiques françaises mises en place depuis la fin des années 90 pour améliorer la sélection et l'admission des candidats des études en France ont-elles changé les tendances déjà évoquées? L'enquête de 2005 de l'OVE sur les étudiants étrangers en France nous apporte quelques éléments de réponse. 


\section{LE PROJET D'AVENIR DES ÉTUDIANTS ÉTRANGERS EN FRANCE}

Les résultats de l'enquête de l'OVE nous permettent de mieux cerner les caractéristiques les plus importantes des étudiants étrangers inscrits dans les universités françaises, à partir de quatre informations majeures :

- Le rapport à la langue française : quatre étudiants sur cinq appartiennent à l'espace francophone (français langue maternelle, langue d'enseignement ou langue utilisée en famille). Un étudiant étranger sur cinq a appris la langue française comme langue étrangère en dehors de l'école ou en auto-formation.

- Le choix de la France comme pays d'études : pour $81 \%$, la France a constitué le premier choix et $19 \%$ auraient préféré étudier dans un autre pays. « Recevoir une formation de qualité » est l'attente la plus importante $(72 \%)$ exprimée par les étudiants, suivie par les réponses sur la «connaissance d'un autre pays et avoir une expérience étrangère » $(44 \%)$ et les attentes pour « faire de la recherche » $(32 \%)$. Ces attentes sont cohérentes avec les tendances observées dans les motivations. Deux grandes tendances se dégagent : la première liée à une logique de formation et de recherche, la seconde s'inscrit plutôt dans une perspective plus culturelle et de découverte. - Le souhait de compléter ses études: La majorité des étudiants étrangers de l'enquête a obtenu déjà un premier diplôme supérieur dans son pays d'origine et son voyage en France vise à compléter ses études. Près d'un étudiant sur quatre commence ses études universitaires en France et un sur dix se réoriente vers une filière différente de celle étudiée dans son pays.

- La venue dans le cadre d'une initiative individuelle : la majorité des étudiants étrangers vient en France dans le cadre d'une initiative individuelle sans bénéficier d'une aide financière institutionnelle permettant de financer entièrement les études. Trois sources financières sont importantes: la famille, l'activité rémunérée ${ }^{6}$, et les différentes aides publiques. Près de deux étudiants sur trois doivent travailler d'une façon régulière $(40 \%)$ ou occasionnelle $(20 \%)$ pour financer leurs études, et près d'un étudiant sur deux est aidé par sa famille.

\footnotetext{
${ }^{6}$ Les étudiants étrangers inscrits à l'université peuvent exercer une activité professionnelle à mi-temps après avoir obtenu une autorisation préalable.
}

\section{Graphique 1 \\ Le projet $d$ 'avenir des étudiants selon les régions d'origine des étudiants}

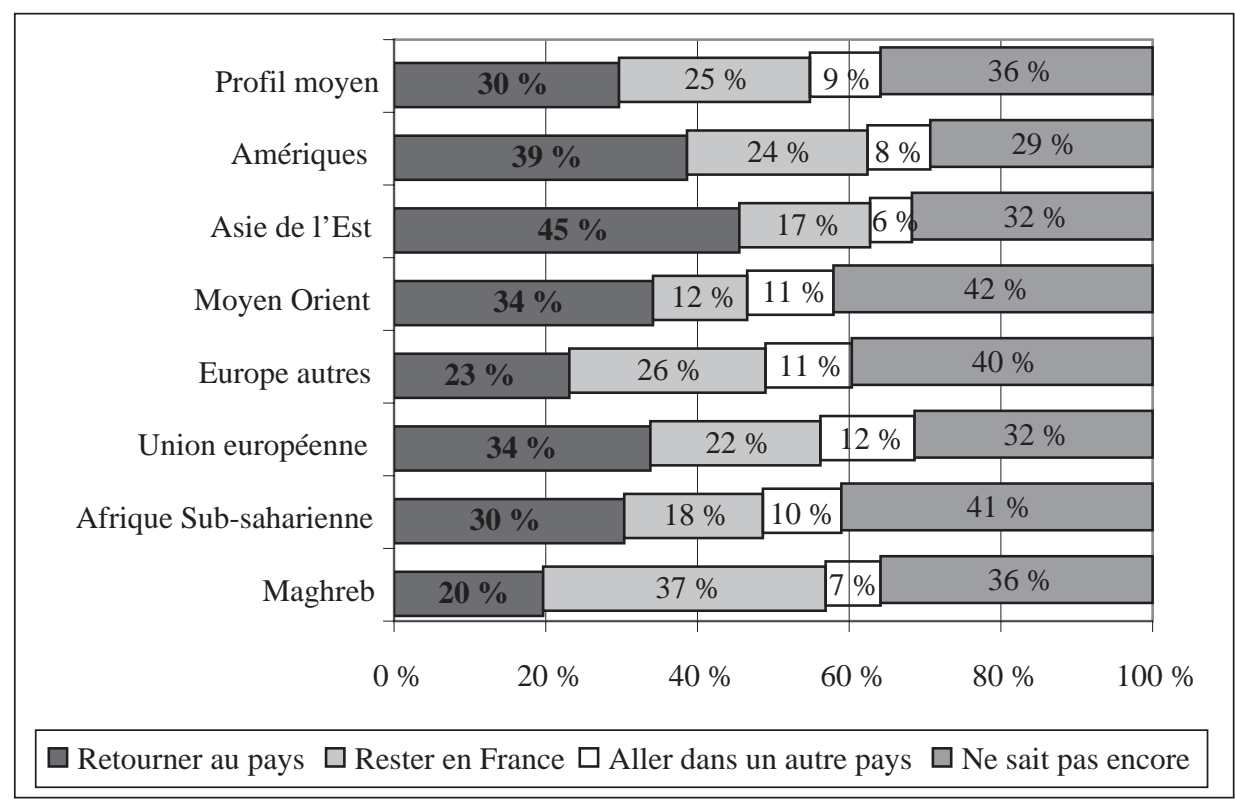

Lecture : $20 \%$ des étudiants maghrébins pensent retourner au pays après la fin de leurs études en France. Source : Enquête 2005 - OVE. 
L'enquête a proposé une série de questions relatives au projet d'avenir à la fin des études afin de mieux connaitre et comprendre les éléments en jeu autour de la question du retour. À la question «Que pensezvous faire après vos études? » $36 \%$ déclarent ne pas le savoir encore, plus d'un tiers (36\%) ne pense pas rentrer $(25 \%$ pensent rester en France et $9 \%$ souhaitent aller dans un autre pays). Enfin, seulement $30 \%$ envisagent de retourner au pays. Deux chiffres sont surprenants: le faible taux de retour prévu d'une part et la proportion élevée des étudiants indécis d'autre part. Ces données montrent que le non-retour est un véritable phénomène chez les étudiants étrangers qui traversent un moment d'incertitude pesant lourdement sur leur projet.

Un premier examen des projets futurs selon les régions d'origines révèle déjà des différences très significatives : «retourner au pays » est un projet plus prégnant pour les étudiants originaires des régions de l'Asie de l'est (45\%), des Amériques (39 \%), du Moyen-Orient (34 \%) et de l'Union européenne $(34 \%)$. Rester en France est plus partagé parmi les Maghrébins (37\%), suivis par les étudiants européens (hors l'UE), $26 \%$ de cette population pensant rester en France. Quant aux indécis, ils sont plus nombreux parmi les étudiants du Moyen-Orient (42\%), de l'Afrique sub-saharienne (41\%) et des autres pays européens $(40 \%)$. Ces résultats sont également assez surprenants dans la mesure où aucune tendance marquée ne domine dans une région. On s'attendait à voir les étudiants du Sud exprimer leur souhait de rester et les étudiants du
Nord prêts à repartir chez eux. Or, les tendances sont loin d'être aussi nettes et divergentes.

Les données de l'enquête montrent que les étudiants ne se différencient pas d'une manière notable selon le sexe, l'âge et le niveau d'études des parents à propos de leur projet d'avenir. Néanmoins, si on distingue les régions d'origine, celles-ci caractérisent plus ou moins fortement les «types» (typologie) d'étudiants que nous définissons plus loin. Parmi les boursiers, il est assez significatif de retrouver plus de candidats pour le retour dans le pays, ils sont deux fois plus nombreux que la moyenne, notamment pour les boursiers Erasmus ; les moins nombreux proportionnellement parmi les boursiers à souhaiter rentrer dans leur pays sont les boursiers du gouvernement français ${ }^{7}$.

Par ailleurs, il existe des différences notables quand on observe les disciplines d'études associées à ces décisions ( $C f$ tableau 1) : «Retourner au pays » apparaît plus associé aux disciplines des sciences économiques et du droit, "Rester en France » aux sciences et techniques et pour les « indécis » (ne sait pas encore) aux lettres, art et langues. Ces réponses montrent que le projet de l'étudiant prendrait en considération certains facteurs comme la situation du marché du travail dans le pays d'origine car le clivage observé entre les filières laisse à penser que les perspectives professionnelles pèsent sur le projet actuel.

${ }^{7}$ Le gouvernement français accorde chaque année une série de bourses aux candidats d'études en France. Les pays africains sont les principaux bénéficiaires de ces bourses d'études.

Tableau 1

Le projet $d$ 'avenir des étudiants étrangers selon les disciplines d'études en France (en \%)

\begin{tabular}{|l|c|c|c|c|c|c|}
\hline & $\begin{array}{c}\text { Retourner } \\
\text { au pays }\end{array}$ & $\begin{array}{c}\text { Rester } \\
\text { en France }\end{array}$ & $\begin{array}{c}\text { Aller dans } \\
\text { un autre pays }\end{array}$ & $\begin{array}{c}\text { Ne sait pas } \\
\text { encore }\end{array}$ & Total & Effectifs \\
\hline Sciences et techniques & 25 & 32 & 9 & 34 & $100 \%$ & 572 \\
\hline Sciences sociales et humaines & 31 & 23 & 9 & 37 & $100 \%$ & 332 \\
\hline Lettres, arts, langues & 27 & 23 & 9 & 40 & $100 \%$ & 284 \\
\hline Sciences éco et droit & 33 & 20 & 11 & 36 & $100 \%$ & 405 \\
\hline Profil moyen & 30 & 25 & 9 & 36 & $100 \%$ & 1593 \\
\hline
\end{tabular}

Khi2 $=22,8$.

$p=0,007$ (Très significatif), non réponses exclues $=122$.

Source : Enquête 2005 - OVE. 
Les réponses fournies à propos de la possibilité de trouver un travail après les études semblent aller dans le sens de cette hypothèse, sans pour autant constituer l'unique explication ( $C f$. tableau 2). Trouver facilement du travail dans son pays après les études est une idée partagée par moins d'un tiers des étudiants (31\%), la majorité pense que ce sera plus ou moins facile (54\%) et $15 \%$ plutôt difficile. Dans l'ensemble, ils anticipent un avenir professionnel relativement mitigé et la représentation qu'ils en ont participerait au renforcement de l'incertitude qui règne dans l'esprit des étudiants étrangers. Selon les filières d'études suivies en France, le sentiment sur les possibilités de trouver du travail dans le pays d'origine apparaît aussi donc assez contrasté comparé au profil moyen: les plus «optimistes» sont les étudiants inscrits dans les disciplines scientifiques et techniques, et les plus «pessimistes » ceux inscrits en sciences humaines et sociales; entre les deux, ceux qui «doutent» sont les étudiants en lettres, arts, langues, sciences économiques et droit.

Ces différences observées selon les disciplines le sont également quand elles se combinent avec le cycle d'études. Les inscrits en doctorat des sciences (44\% pensent trouver facilement un travail après la fin des études) sont logiquement plus optimistes que ceux qui sont inscrits au niveau du doctorat en lettres, arts et langues (19\%), de la licence de ces filières ( $23 \%)$, du doctorat ou de la licence des sciences sociales et humaines (respectivement $27 \%$ et $24 \%$ ). Les étudiants inscrits en sciences économiques et en droit occupent une position intermédiaire quant à leur chance de trouver facilement un travail : $33 \%$ en licence et $37 \%$ en doctorat. Malgré la proportion plus élevée des étudiants inscrits en doctorat qui pensent trouver un emploi, les distinctions uniquement par niveau ne sont pas significatives, l'effet du niveau n'étant pas le même selon les disciplines.

En distinguant l'origine géographique des étudiants, on note des variations qui peuvent être liées certainement aux situations du marché du travail de leur pays d'appartenance. Ainsi, les étudiants originaires de l'Europe (hors de l'UE), du Moyen Orient et des Amériques apparaissent un peu moins "pessimistes » que les autres; ils pensent plus fréquemment qu'après leurs études il sera facile de trouver du travail dans leur pays. À l'opposé, les étudiants issus des pays de l'Union européenne et du Maghreb sont plus nombreux que les autres à penser pouvoir « difficilement » trouver du travail.

Comme l'illustre le tableau 2, le fait de penser intégrer facilement ou difficilement le marché du travail dans son pays est statistiquement lié aux décisions à prendre après les études : $36 \%$ des personnes qui estiment pouvoir trouver «facilement» un travail dans le pays envisagent d'y retourner contre seulement $18 \%$ pour ceux qui pensent le contraire. Ces proportions s'inversent lorsqu'il s'agit d'envisager de «rester en France » : $22 \%$ qui pensent trouver « facilement » un emploi à la fin des études dans leur pays comptent rester en France contre $39 \%$ des étudiants pour lesquels la perspective professionnelle est « difficile». Cependant, comme en témoigne le tableau 2, l'emploi ne constitue pas le seul facteur d'appréciation des étudiants interrogés quant à la question du retour, notamment pour les indécis.

Tableau 2

Le projet d'avenir des étudiants étrangers selon la possibilité de trouver un travail dans le pays d'origine (en \%)

\begin{tabular}{|l|c|c|c|c|c|c|}
\hline & $\begin{array}{c}\text { Retourner } \\
\text { au pays }\end{array}$ & $\begin{array}{c}\text { Rester } \\
\text { en France }\end{array}$ & $\begin{array}{c}\text { Aller dans } \\
\text { un autre pays }\end{array}$ & $\begin{array}{c}\text { Ne sait pas } \\
\text { encore }\end{array}$ & Total & Effectifs \\
\hline Facilement & 36 & 22 & 8 & 34 & $100 \%$ & 505 \\
\hline Plus ou moins facilement & 29 & 23 & 9 & 38 & $100 \%$ & 884 \\
\hline Difficilement & 18 & 39 & 11 & 32 & $100 \%$ & 248 \\
\hline Profil moyen & 30 & 25 & 9 & 36 & $100 \%$ & 1637 \\
\hline
\end{tabular}

Khi2 $=44,2$.

$p=0,001$ (Très significatif), non réponses exclues $=78$.

Source : Enquête 2005 - OVE. 
Tableau 3

Le projet d'avenir des étudiants étrangers selon leur projet initial (en \%)

\begin{tabular}{|l|c|c|c|c|c|c|}
\hline & $\begin{array}{c}\text { Retourner } \\
\text { au pays }\end{array}$ & $\begin{array}{c}\text { Rester } \\
\text { en France }\end{array}$ & $\begin{array}{c}\text { Aller dans } \\
\text { un autre pays }\end{array}$ & $\begin{array}{c}\text { Ne sait pas } \\
\text { encore }\end{array}$ & Total & Effectifs \\
\hline Compléter des études & 33 & 24 & 8 & 35 & $100 \%$ & 1063 \\
\hline Commencer une formation & 26 & 26 & 12 & 37 & $100 \%$ & 419 \\
\hline Se réorienter & 18 & 23 & 12 & 47 & $100 \%$ & 121 \\
\hline Total & 30 & 25 & 9 & 36 & $100 \%$ & 1603 \\
\hline
\end{tabular}

Khi2 $=21$.

$p=0,002$ (Très significatif), non réponses exclues $=112$.

Source : Enquête 2005 - OVE.

Confronté au projet initial, avant de venir en France, la question du retour différencie de façon significative les réponses des étudiants étrangers de notre enquête ( $C f$. Tableau 3). Le sentiment d'incertitude est significativement plus présent parmi les étudiants qui avaient comme projet initial de se réorienter $(47 \%$ ne savent pas encore ce qu'ils vont faire). "Retourner au pays » est plus fréquemment évoqué par ceux qui sont venus compléter leurs études $(33 \%)$ et «aller dans un autre pays » est plus souvent exprimé par ceux (les plus jeunes) qui sont venus commencer des études (12\%). À l'opposé, les étudiants qui sont en réorientation sont proportionnellement moins nombreux à vouloir rentrer au pays (18\% contre $30 \%$ pour l'ensemble). Ce qui expliquerait éventuellement la raison de leur réorientation.

L'enquête demandait également aux étudiants interrogés de préciser s'ils pensaient rester en France et les raisons de cette décision. Les 408 étudiants souhaitant rester en France évoquent en premier lieu les raisons professionnelles ( $52 \%$ ), viennent ensuite les raisons familiales ( $32 \%$ ) et enfin les raisons politiques, culturelles et sociales qui sont citées par $16 \%$ des étudiants. Ces chiffres témoignent que, en dehors des raisons familiales, c'est surtout la situation professionnelle qui peut influencer la décision relative au retour ou au non-retour.

Nous avons tenté d'étudier les liens statistiques significatifs entre certaines variables concernant le moment du départ de l'étudiant à l'étranger (projet initial, parcours antérieur) et le moment de l'enquête à l'aide d'une analyse factorielle ${ }^{8}$. Le tableau 4 en présente une synthèse ainsi que la typologie élaborée. Deux sous-populations d'étudiants sont identifiées : la première est regroupée dans le «Type $\mathrm{A}$ » et la seconde comprend les « Type B, C, D et E »). Cette classification distingue les étudiants selon les caractéristiques dominantes suivantes :

Le « Type A» (298 étudiants, $17 \%$ de la population totale) regroupe surtout des étudiants venus " commencer » des études supérieures $(82 \%)$; parmi eux, les étudiants originaires d'Afrique sub-saharienne ( $60 \%$ du total de cette région), les plus « indécis » visà-vis de leur avenir, y sont surreprésentés (1,2 fois) et ceux qui pensent également aller dans un autre pays. Plus des trois quarts de leurs parents (père et/ou mère) ont un niveau d'études supérieures.

Les « Types B-C » et les « Types D-E » regroupent le reste de l'échantillon (1 417 étudiants) avec les regroupements et les caractéristiques dominantes suivantes :

- Le «Type B» (368 étudiants) se caractérise par une forte présence d'étudiants venus compléter leurs

${ }^{8}$ La classification ou la typologie a été obtenue à partir d'une procédure de calcul sur les résultats d'une analyse factorielle multiple et d'une analyse discriminante, les deux premiers axes représentaient un plan de $40 \%$ de l'inertie totale. Les variables qui ont contribué à l'analyse factorielle sont les suivantes : le projet initial, le projet d'avenir, les attentes vis-à-vis des études supérieures en France, les régions d'origine, la discipline d'études, les motivations concernant le choix de la France comme pays d'études, le bilan des études, le sexe et le niveau d'études des parents. La typologie établie doit être comprise comme une approximation de " groupes d'individus " construite à partir de la présence simultanée des variables. L'analyse de correspondances multiples nous propose donc les regroupements qui ne sont pas des types à l'état pur, mais des types à l'état approché. 
Tableau 4

Le profil des «Types » selon le projet à la fin des études

\begin{tabular}{|l|c|c|c|c|c|c|c|}
\hline & $\begin{array}{c}\text { Retourner } \\
\text { au pays }\end{array}$ & $\begin{array}{c}\text { Rester en } \\
\text { France }\end{array}$ & $\begin{array}{c}\text { Aller dans un } \\
\text { autre pays }\end{array}$ & $\begin{array}{c}\text { Ne sait pas } \\
\text { encore }\end{array}$ & Total & Effectifs & En \% Colonne \\
\hline Type A & $28 \%$ & $17 \%$ & $13 \%$ & $42 \%$ & $100 \%$ & $\mathbf{2 9 8}$ & $17 \%$ \\
\hline Type B & $18 \%$ & $44 \%$ & $5 \%$ & $33 \%$ & $100 \%$ & $\mathbf{3 6 8}$ & $21 \%$ \\
\hline Type C & $25 \%$ & $26 \%$ & $10 \%$ & $38 \%$ & $100 \%$ & $\mathbf{4 0 4}$ & $24 \%$ \\
\hline Type D & $37 \%$ & $15 \%$ & $11 \%$ & $37 \%$ & $100 \%$ & $\mathbf{3 4 4}$ & $20 \%$ \\
\hline Type E & $\mathbf{4 0 \%}$ & $23 \%$ & $7 \%$ & $30 \%$ & $100 \%$ & $\mathbf{3 0 1}$ & $18 \%$ \\
\hline Profil Moyen & $\mathbf{3 0} \%$ & $\mathbf{2 5} \%$ & $\mathbf{9} \%$ & $\mathbf{3 6} \%$ & $\mathbf{1 0 0} \%$ & $\mathbf{1 7 1 5}$ & $\mathbf{1 0 0} \%$ \\
\hline
\end{tabular}

Lecture : $40 \%$ des étudiants appartenant au type E pensent retourner au pays. Source : Enquête 2005 - OVE.

études ( $88 \%$ du total de cette catégorie) ; il est composé de plus de la moitié des étudiants maghrébins (53\%) qui représentent plus de $80 \%$ des « classés » dans cet ensemble B; $63 \%$ sont des étudiants en sciences et techniques, $88 \%$ souhaitent obtenir un doctorat et les trois quarts souhaitent faire de la recherche, $44 \%$ pensent rester en France après leurs études, un tiers ne sait pas encore. Plus des deux tiers sont des femmes et plus des deux tiers (67\%) ont des parents ayant un niveau d'études inférieur à l'enseignement supérieur.

- Le «Type $C$ » (404 étudiants) comprend $58 \%$ d'étudiants venus compléter des études et $32 \%$ les commencer; après les études, $38 \%$ ne savent pas encore ce qu'ils feront, un quart pense retourner au pays, un autre quart pense rester en France et $10 \%$ pensent à un autre pays (assez proche du profil moyen). Comme pour le type précédent $(\mathrm{C})$, plus des deux tiers sont des femmes. Ce type est composé surtout d'étudiants originaires du Maghreb $(46 \%)$ et d'Afrique Sub-saharienne (37\%). Près de $60 \%$ des étudiants appartenant à ce type ont des parents ayant un niveau d'études inférieur à l'enseignement supérieur.

Ces trois types (A, B et C) regroupent presque la totalité des étudiants maghrébins et africains (subsahara).

- Le «Type D» (344 étudiants), se caractérise par les traits dominants suivants: $59 \%$ sont venus compléter leurs études. Connaître un autre pays, avoir une expérience étrangère et recevoir une for- mation de qualité constituent leurs attentes majeures (96\%), un petit effectif (4\%) vise l'obtention d'un master pour faire une thèse et de la recherche; ils visent plutôt un Master 2. Le taux de souhait de retour au pays après les études est supérieur à la moyenne $(37 \%)$, mais avec un taux «d'indécis» aussi important. Les origines géographiques les plus représentées sont l'Europe (l'UE et les autres pays européens, $50 \%$ et $54 \%$ respectivement des populations de ces régions), $26 \%$ des étudiants sont originaires de l'Asie de l'Est, (45\% du total des étudiants de cette région), $39 \%$ des étudiants des Amériques ; enfin, $80 \%$ sont des étudiantes.

- Le «Type E» (301 étudiants) se distingue du précédent plus particulièrement par le diplôme visé, le Doctorat. Aux précédentes origines s'ajoutent les étudiants originaires du Moyen-Orient ( $46 \%$ du total des étudiants de cette région). C'est parmi ces étudiants que le taux de retour au pays est le plus élevé ( $40 \%$, soit près d'une fois et demi plus élevée que la moyenne).

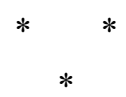

Les données statistiques issues de l'enquête par questionnaire, comme les propos des étudiants lors des entretiens semi-directifs - centrés sur la vie privée, le vécu en tant qu'étudiant étranger, la famille...participent au renouvellement des interrogations sur le sens de la mobilité internationale des étudiants et 
de leur projet d'avenir. Dans leur ensemble, ils tendent à confirmer une grande diversité de comportements, et la présence simultanée et interactive des facteurs structurels (le marché du travail, les conditions de vie) et des situations individuelles. Une incertitude existe aussi quant au projet d'avenir des étudiants étrangers. Elle concerne toutes les régions sans que les motifs soient forcément les mêmes. D'autres enquêtes sur les étudiants étrangers avaient également mis en évidence qu'une partie importante d'entre eux n'envisageaient pas de rentrer dans leur pays, tout au moins immédiatement après la fin de leurs études, et cherchaient une installation définitive ou une insertion provisoire sur le marché du travail du pays d'accueil.

Ainsi, les réponses à la question relative aux raisons du non-retour montrent bien que le projet d'avenir des étudiants ne s'inscrit pas uniquement dans une logique marchande. Le non-retour n'a pas seulement une perspective économiste ou mécaniste. Les étudiants qui, à travers des trajectoires et des projets, vivent des situations assez complexes, doivent souvent bricoler pour atteindre leurs objectifs ou les ajuster. De même, leurs réponses nuancées résultent des incertitudes inhérentes au voyage éducatif, qui est de fait une migration temporaire. Les entretiens semi-directifs réalisés auprès d'une partie des étudiants de notre échantillon montrent que l'étudiant semble se trouver face à des perspectives qui ne sont pas rassurantes : le doute est partout, ici et là-bas, ou ailleurs. Le retour suscite des craintes, mais rester dans le pays d'accueil aussi.

L'enquête (le questionnaire et les entretiens) a permis de repérer plusieurs figures d'étudiants étrangers n'ayant pas un projet de retour lors de l'enquête. Ces figures se construisent à travers les logiques sous-jacentes des stratégies mises en œuvre par les étudiants étrangers à propos de leur avenir. Les logiques identifiées semblent interagir et se combiner dans un processus réflexif: il s'agit des logiques professionnelle, migratoire, intégratrice et existentielle.

Tout d'abord, on observe une logique professionnelle, inhérente à toute entreprise d'études ou de formation. Le projet d'aller dans un pays étranger s'organise souvent dans le but d'acquérir un maximum de connaissances ou un diplôme plus élevé ou mieux valorisé. Il s'agit de mettre toutes les chances de son côté afin de réussir une insertion professionnelle. L'émigration, qui est motivée par la recherche d'une bonne formation, se prolonge, pour beaucoup de diplômés étrangers, par la recherche d'emploi en dehors du pays d'origine. Les diplômés cherchent à mettre ainsi à leur profit ce nouvel espace professionnel ouvert et «internationalisé ». Le marché du travail des pays développés suscite et encourage certains non-retours, compte tenu des besoins locaux. La motivation professionnelle pour rester en France ne semble pas s'expliquer uniquement par le manque de débouchés dans le pays d'origine, mais aussi parce qu'ici certains ont déjà rencontré de meilleures conditions de travail. Le nonretour a parfois un caractère « provisoire", quand il s'agit d'acquérir une première expérience professionnelle en Europe en attendant le retour. Certains étudiants, dans leur façon de penser leur devenir, mettent en avant un projet d'emploi en France pour différer ainsi le retour.

La seconde logique est migratoire et la stratégie d'installation est conçue dès le départ (voire même avant celui-ci). Le réseau de parents et d'amis étend la dispersion familiale et facilite l'installation dans un autre pays. De même, l'existence d'une communauté fortement implantée génère une dynamique migratoire et les études à l'étranger servent d'étape intermédiaire. L'espace migratoire s'organise souvent à partir des liens multiples qui relient migrants et futurs migrants. Le fait qu'un nombre non négligeable d'étudiants étrangers mentionnent leurs points d'attache familiaux révèle que la France constitue un choix «naturel» pour eux. Les entretiens montrent que cette logique est présente très souvent avant le départ vers la France dans le cas des étudiants appartenant aux principales communautés immigrées en France (les pays africains).

On observe également une logique liée à la dynamique intégratrice, avec ses dimensions affectives, sociales et culturelles, qui peut contribuer à une installation non prévue. Comme on a pu l'observer à travers les entretiens, l'étudiant étranger vit une vraie socialisation «secondaire » en bravant l'extérieur et en bifurquant ailleurs, un processus d'acculturation permettant d'explorer de nouveaux liens sociaux et 
de développer les modes d'approche de l'altérité. Les étudiants étrangers travaillent, étudient, font des recherches, participent aux activités culturelles, vivent leur affectivité et consomment. Dans cette perspective, le voyage éducatif peut participer à la transformation de leur identité culturelle et à l'évolution de leurs «perspectives ». Cette logique met en avant le caractère interactif et transformateur du voyage, le devenir n'est pas donné, il est construit chemin faisant. Comme en témoignent les entretiens menés, une cohabitation réussie, une rencontre amoureuse, de meilleures conditions de vie peuvent donner naissance à un projet d'installation provisoire ou définitive.

Enfin, il existe une logique existentielle qui concerne les étudiants, souvent des femmes, originaires de pays où ils se sentent moins libres. Rester en France participe à cette reconstruction intellectuelle et à ce « bonheur intime » engendrés par le voyage éducatif, à l'expérience de liberté, ou à l'épanouissement culturel. Certains étudiants sont prêts à accepter un emploi sans rapport avec leur qualification afin de bénéficier d'un cadre de vie convenable, ou pour fuir l'humiliation; c'est le cas des étudiants issus de certains pays du Sud.

Ces quatre logiques construites en combinant les données «qualitatives» (issues des entretiens) et statistiques doivent être considérées comme une autre lecture des réponses fournies par les étudiants à propos de leur projet d'avenir. Elles peuvent diversement concerner les cinq groupes identifiés dans la typologie élaborée par l'analyse factorielle. Ainsi, par exemple, la logique professionnelle semble concerner davantage les types $\mathrm{B}$ et $\mathrm{C}$, la logique migratoire les type $\mathrm{B}$ et $\mathrm{C}$, la logique intégratrice les types A et D et la logique existentielle le type D.

Ces quatre logiques liées au phénomène du nonretour ne se produisent pas par attraction mécanique depuis un «centre», ou en empruntant seulement un mode de calcul rationnel. Elles n'obéissent pas non plus à une parfaite linéarité ou à une sur-détermination d'une dynamique de marché. Voyager à l'étranger met l'étudiant dans une situation de transition: les transformations identitaires vécues peuvent entraîner des remises en cause parfois radicales des projets initiaux et des représentations. La satisfaction des études ou la réussite universitaire n'expliquent pas à elles seules le projet de retour des étudiants: malgré les plaintes récurrentes sur les conditions d'études ou de vie (logement, financement d'études, démarches administratives harassantes), la grande majorité des étudiants interrogés dans l'enquête est satisfaite de son vécu en France et n'a pas l'intention de rentrer au pays. La mobilité devient un modus vivendi (Nedelcu, 2004, p. 12), la population étrangère des universités représente des réalités multiples et parfois contradictoires: les "artisans des mondes vécus dépourvus de distances » (Beck, 2001), les «nomades » du savoir (Meyer, 2001), les « oiseaux migrants» (MurphyLejeune, 1998) ou les déboussolés ou les bohêmes en quête de sens ou d'un port d'ancrage. La fuite ou la circulation des cerveaux doit être aujourd'hui comprise à travers la complexité des situations individuelles et l'impact des données structurelles (marché du travail, conditions de vie et de travail).

La théorie de l'exode des cerveaux et celle relative à la circulation des cerveaux ne semblent pas être tout à fait adaptées pour expliquer toute la complexité du phénomène du non-retour des étudiants étrangers. L'enquête révèle l'existence d'une véritable filière d'immigration par le biais des études à l'étranger. Celle-ci revêt plusieurs facettes. On ne peut pas la considérer systématiquement comme un exode des cerveaux. Seule une partie des étudiants qui tentent de rester dans le pays d'accueil entre dans la logique de l'exode des cerveaux. Le non-retour concerne des étudiants ayant des niveaux et des compétences très variés, pas toujours en adéquation avec le marché du travail du pays d'accueil. 


\section{Bibliographie}

Agarwal. V. B. et Winkler D. R. (1985a), «United States Immigration Policy and Indirect Immigration of Professionals », Economics of Education Review 4, $\mathrm{n}^{\circ} 1$, pp. 1-16.

Agarwal V. B. et Winkler D. R. (1985b), « Migration of Foreign Students to the United States », Journal of Higher Education, ${ }^{\circ}$ 56, pp. 509-522.

Agarwal V. B. et Winkler D. R. (1984), «Migration of Professional Manpower to the United States ", Southern Economic Journal, Vol. 50, n 3, pp. 814830.

Banque mondiale (2004), Perspectives pour l'économie mondiale, Washington: Banque mondiale.

Beck U. (2001), La société du risque. Sur la voie d'une autre modernité, Paris : Alto Aubier.

Behnam D. (1981), Porteur de connaissances, étudiant et expert étranger et échange de connaissances pour un développement endogène, Paris, UNESCO.

Ben Sedrine S. et Geisser V. (2001). Le retour des diplômés. Enquête sur les étudiants tunisiens formés à l'étranger. Europe, Amérique et monde arabe, Tunis, Centre des Publications Universitaires (CPU).

Benguerna M. et Khelifaoui H. (1993), Bilan et réinsertion des boursiers algériens à l'étranger relevant $d u$ Ministère de l'enseignement supérieur, Alger, CREAD et MEN Algérie.

Bhagwati J. N. (1976), The Brain Drain and Taxation, New York: North Holland Publishing Compagny.

Borgogno V. et Vollenweider-Andresen L. (1998), «Les étudiants étrangers en France: trajectoires et devenir », Migrations Études, $n^{\circ} 79$.
Boussichas M. (2005), Une fuite des cerveaux bénéfique existe t-elle? (Texte diffusé sur Internet : http :/ /team.univ-paris1.fr/teamperso/DEA/Actualites/ Doctoriales/M.Boussichas.pdf).

Cervantes M. et Guellec D. (2002), «Fuite des cerveaux: Mythes anciens, réalités nouvelles », $L^{\prime} O b s e r v a t e u r$ de l'OCDE, $\mathrm{n}^{\circ} 230$.

Coulon A., Paivandi S. (2003), Les étudiants étrangers en France : l'état des savoirs, Rapport à l'OVE national.

Douieb El Attafi A. (1989), Étude de la perspective de retour, non-retour chez les étudiants maghrébins (retours, non-retours des étudiants marocains: le cas de Lille), Thèse de doctorat, université Lille 1.

Gaillard A.-M. et Gaillard J. (1999), Les enjeux des migrations scientifiques internationales. De la quête du savoir à la circulation des compétences, Paris, L'Harmattan.

Glaser A. W. (1978), The brain drain: Emigration and retrun. New York : Pergamon Press.

Glaser A. W. (1973), The Migration and return of professionnels, Colombia University, Bureau of applied Social Research.

Gossman C. S. and al. (1968). Migration of college and University Students in the United States, Seattle : University of Washington Press.

Kim S. M. (2000), Les femmes asiatiques et l'enseignement supérieur en France : rapport au savoir et positionnement social dans les sociétés asiatiques (Corée du Sud, Japon et Tä̈wan), Thèse de doctorat, université Paris 8.

Latrèche A et Geisser V. (2001), « Faire ses études en France et après? Le devenir des étudiants maghrébins formés dans l'Hexagone », Migration Société, vol. $13, \mathrm{n}^{\circ} 74$, pp. 87-97. 
Latrèche A. (1999), La migration internationale des étudiants: le cas des étudiants maghrébins en France, Thèse de $3^{\mathrm{e}}$ cycle, université Paris 1.

Meyer J.-B. et Hernandez V. (2004), « Les diasporas scientifiques et techniques: état des lieux », in Nedelcu M. (dir.) La mobilité internationale des compétences: situations récentes approches nouvelles, Paris, L'Harmattan.

Meyer J.-B. (2001), «Fuite des cerveaux: les nouveaux termes de l'exode ", Le Courrier ACP-UE, Juillet-Aout.

Meyer J.-B. et Charum J. (1995), La «fuite des cerveaux" est-elle épuisée? Paradigme perdu et nouvelles perspectives, Paris, IRD.

Michaelis A. (1990), "Brain drain and brain gain », Interdisciplinary Science Reviews, $\mathrm{n}^{\circ}$ 15(3).

Murphy-Lejeune E. (1998). L'étudiant européen voyageur, un nouvel «étranger ». Aspects de l'adaptation interculturelle des étudiants européens, Thèse de doctorat, université Nancy 2.

Naraghi E. (1965), Le problème de l'émigration des scientifiques et des techniciens (l'exode des compétences ou « brain drain »), Paris, UNESCO.

Nedelcu M. (2004) (dir.), La mobilité internationale des compétences: situations récentes approches nouvelles, Paris, L'Harmattan.

OCDE (2006), Perspectives des migrations internationales, SOPEMI (Système d'observation permanente des migrations), Paris, OCDE.

OCDE (2003), Regard sur l'Éducation 2002, Paris.

OCDE (2002), International Mobility of the Highly Skilled, Paris.

OCDE (1998), Tendances des migrations internationales, Paris.
Orivel F. (1991), « La crise des universités francophones sub-saharienne », Perspectives, UNESCO, $\mathrm{n}^{\circ} 79$.

Paivandi S. (1991), Les étudiants iraniens en France. Le cas de l'Université Paris VIII, Thèse de doctorat, université Paris 8.

Raunet M. (2001), De l'exode à la mobilisation des compétences dans le cadre d'un véritable co-développement, Conseil économique et social, Paris, Éditions des Journaux Officiels.

Rovet M., Terouanne D., Neher E. (1998), Higher Education in France and the International Migration of Scientists, Mimeo, CNRS Washington Office.

Salomon J.-J. (1991), « Brain drain, les logiques de l'exode ", in Witkowski N. (dir.), L'état des sciences et des techniques, Paris, La Découverte.

Sefrioui S. (1997). Les migrations étudiantes des pays en développement vers les pays développés: efficience et équité. Étude dans le cas particulier des flux d'étudiants vers la France, Thèse de doctorat, université Dijon.

Simon V. (1997). La migration des étudiants maghrébins en France et ses transformations (19621994), Thèse de doctorat, université Paris 7.

Vuilletet G. (2005), Comparaison internationales des politiques d'accueil des étudiants étrangers : Quelles finalités? Quels moyens?, Rapport du Conseil économique et social.

Wickramasekara P. (2003), Options politiques de réponse à la migration des compétences : rétention, retour et circulation, Genève : Bureau International du Travail.

Zaho J. (2000), «Brain drain and brain gain: The migration of knowledge workers from and to Canada », Education quarterly review, $\mathrm{n}^{\circ} 3$. 


\title{
Résumé
}

\section{Le non-retour des étudiants étrangers : au-delà de la «fuite des cerveaux »}

\author{
Ridha Ennafaa et Saeed Paivandi
}

Cet article réexamine la question du retour des étudiants étrangers dans leur pays d'origine après les études. II se fonde sur une enquête de l'Observatoire de la vie étudiante (OVE) réalisée en 2005 auprès de 1715 étudiants étrangers dans dix universités françaises. L'article s'appuie sur une documentation internationale pour montrer, dans une première partie, l'évolution du paradigme de la "fuite des cerveaux», son lien avec les études à l'étranger et ses limites. La deuxième partie est consacrée aux projets d'avenir des étudiants étrangers enquêtés. Cette contribution vise à participer au renouvellement des interrogations, d'une part, sur la signification de la mobilité internationale des étudiants et, d'autre part, sur la question du non-retour face à l'instauration de dispositifs visant à améliorer la sélection et l'accueil des candidats aux études en France.

\section{Mots clés}

Fuite des cerveaux, Étudiant, Population d'origine étrangère, Mobilité internationale, Enquête, France Journal of Economic Literature : F 22, J 24 